\section{Resultados a largo plazo del trasplante intracoronario de células mononucleares de médula ósea autólogas en pacientes con cardiopatía dilatada de diversa etiología}

\author{
JORGE BARTOLUCCI ${ }^{1,2}$, FERNANDO J. VERDUGO ${ }^{1}$, \\ FLAVIO CARRION $^{1}$, EMA ABARZÚA ${ }^{2}$, CARLOS GOSET ${ }^{2}$, \\ RUBÉN LAMICH ${ }^{2,3}$, PATRICIO SANHUEZA ${ }^{4}$, \\ PABLO PEDREROS ${ }^{2,3}$, CAROLINA NAZZAL ${ }^{5}$, \\ MAROUN KHOURY ${ }^{1}$, FERNANDO E. FIGUEROA ${ }^{1}$
}

\section{Long-term results of intracoronary transplantation of autologous bone marrow cells in dilated cardiomyopathy}

Background: Intracoronary delivery of autologous bone marrow mononuclear cells is an interesting therapeutic promise for patients with heart failure of different etiologies. Aim: To evaluate the long-term safety and efficacy of this therapy in patients with dilated cardiomyopathy of different etiologies under optimal medical treatment. Patients and Methods: Prospective, open-label, controlled clinical trial. Of 23 consecutive patients, 12 were assigned to autologous bone marrow mononuclear cell intracoronary transplantation, receiving a mean dose of $8.19 \pm 4.43 \times 10^{6} \mathrm{CD} 34+$ cells. Mortality, cardiovascular readmissions and cancer incidence rate, changes in functional capacity, quality of life questionnaires and echocardiographic measures from baseline, were assessed at long-term follow-up (37.7 \pm 9.7 months) in patients receiving or not the cells. Results: No significant differences were observed in mortality, cardiovascular readmissions or cancer incidence rate amongst groups. An improvement in functional class and quality of life questionnaires in the transplanted group was observed $(p<0.01)$. The treated group showed a non-significant increase in left ventricular ejection fraction at long-term follow-up (from $26.75 \pm 4.85 \%$ to $34.90 \pm 8.57 \%, p=0.059$ compared to baseline). There were no changes in left ventricular volumes. We observed no improvement of these variables in the control group. Conclusions: Intracoronary transplantation of autologous bone marrow mononuclear cells is feasible and safe in patients with dilated cardiomyopathy of diverse etiologies. This therapy was associated to persistent improvements in functional class and quality of life. There was also a non-significant long-term improvement of left ventricular function.

(Rev Med Chile 2015; 143: 415-423)

Key words: Stem cell transplantation, Heart Failure, Dilated Cardiomyopathy, Ventricular Function, Quality of Life.

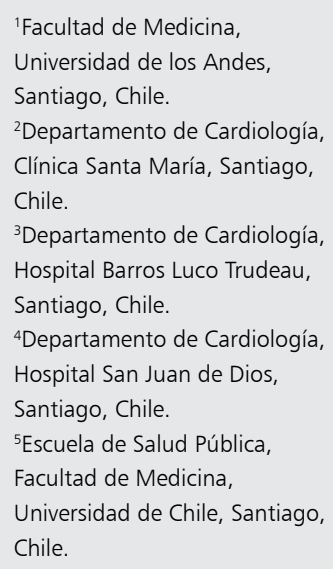

Recibido el 2 de agosto de 2014, aceptado el 15 de enero de 2015.

Fuente de apoyo financiero: Proyecto INNOVA Chile №2054350, CORFO

Conflicts of interest: J. Bartolucci and $\mathrm{M}$ Khoury reported grants from CORFO Innova-Chile during the conduct of the study, personal fees from Cells for CellsUniversidad de los Andes (Chile), and grants from CORFO outside the submitted work.

F. Carrion and F. Figueroa reported grants from CORFO Innova-Chile during the conduct of the study, and grants from CORFO outside the submitted work

Correspondencia a: Dr. Jorge Bartolucci Unidad de Estudios Cardiológicos, Clínica Santa María.

Fernando Manterola 0530, Providencia, Santiago, Chile. jbartolucci@csm.cl. 
E n base a los datos de los estudios clínicos disponibles, el potencial terapéutico de la terapia celular cardiovascular en pacientes con insuficiencia cardiaca crónica, tanto de etiología isquémica como no isquémica, pareciera ser promisorio. Los ensayos clínicos en fase I y II concuerdan en señalar que la administración intracoronaria o intramiocárdica de células madre de diversos orígenes, en pacientes con insuficiencia cardiaca es segura ${ }^{1-7}$. Respecto a la eficacia de esta terapia, se han descrito beneficios significativos sobre la capacidad funcional, la calidad de vida y un aumento de la fracción de eyección del ventrículo izquierdo $(\mathrm{FEVI})^{1-7}$. Pese a sugerir un beneficio importante dentro del primer año, es limitado el número de ensayos con seguimiento prolongado, en pacientes sometidos a terapia celular ${ }^{4-6}$.

En concordancia a lo descrito por otros autores, reportamos en una publicación previa que el trasplante intracoronario de células mononucleares de médula ósea (CMMO) es un procedimiento seguro en pacientes con cardiopatía en fase dilatada de diversa etiología ${ }^{8}$. En términos de eficacia, señalamos un beneficio de esta terapia sobre la función ventricular izquierda y la capacidad funcional de los pacientes tratados versus los controles, luego de 6 y 12 meses de seguimiento ${ }^{8}$.

El objetivo del presente estudio fue evaluar la seguridad y la eficacia del trasplante intracoronario de CMMO en pacientes con cardiopatía en fase dilatada de diversa etiología, en un nuevo corte de seguimiento a largo plazo.

\section{Pacientes y Método}

\section{Población de estudio}

Los participantes fueron referidos al servicio de Cardiología de Clínica Santa María desde el mismo centro y de dos hospitales públicos, entre 2008 y 2011.

Los criterios de inclusión fueron: a) Insuficiencia cardiaca crónica estable en condiciones óptimas de tratamiento, según guías clínicas nacionales ${ }^{9}$; b) FEVI $\leq 40 \%$; c) Edad entre $18 \mathrm{y}$ 75 años; d) Arterias coronarias permeables en pacientes con cardiopatía isquémica crónica y ausencia de lesiones significativas en pacientes con cardiopatía de etiología no isquémica.

Los criterios de exclusión fueron: a) Insuficiencia cardiaca descompensada; b) Taquicardia ventricular; c) Lesión coronaria significativa no resuelta; d) Síndrome coronario agudo durante el último año; e) Fibrilación auricular permanente sin control de frecuencia; f) Enfermedad renal crónica; g) Infección activa; h) Evidencia de patología neoplasia o hematológica; i) Dependencia de alcohol o drogas en los últimos 12 meses.

\section{Diseño}

Ensayo clínico controlado en fase I-II en 23 pacientes con insuficiencia cardiaca crónica en fase dilatada, con disfunción sistólica. El diseño del estudio, incluyendo el seguimiento realizado durante el primer año, esta resumido en la Figura 1 y está descrito en detalle en nuestro reporte previo $^{8}$. Brevemente, los pacientes fueron asignados por orden de ingreso a grupo tratado o control, no realizándose selección por patología de base. Se calculó el tamaño de la muestra considerando un error alfa de $5 \%$, potencia de $90 \%$, estimando una media de FEVI en el grupo trasplantado de $36 \%$ y en el grupo control de $28 \%$, desviación estándar de 4,5\%, necesitándose 7 pacientes por grupo.

Los pacientes del grupo trasplantado fueron sometidos al protocolo de obtención de CMMO y luego a trasplante intracoronario mediante catéter balón, siguiendo los protocolos previamente descritos $^{8}$, recibiendo una dosis media de $1,94 \times 10^{8}$ $\pm 0,53 \times 10^{8}$ CMMO autólogas, para una media de $8,19 \pm 4,43 \times 10^{6}$ células CD34+. Los pacientes del grupo control no fueron intervenidos, decisión respaldada por los Comités de Ética. Todos los pacientes continuaron su tratamiento farmacológico óptimo siguiendo las guías nacionales de tratamiento de insuficiencia cardiaca ${ }^{9}$. El diseño experimental fue aprobado por los Comités de Ética de la Clínica Santa María y del Servicio Metropolitano de Salud Oriente. Todos los pacientes firmaron el consentimiento informado para participar en el estudio.

Como criterio de valoración de seguridad a largo plazo se evaluó la incidencia de eventos clínicos mayores ocurridos desde el primer día hasta el final de seguimiento, incluyendo: mortalidad general y cardiovascular, hospitalizaciones por patología cardiovascular (insuficiencia cardiaca descompensada, síndrome coronario agudo, síncope o accidente cerebrovascular), implante de desfibrilador automático, resincronizador o marcapasos, aparición de neoplasias. El principal criterio de valoración de eficacia fue la FEVI, sien- 


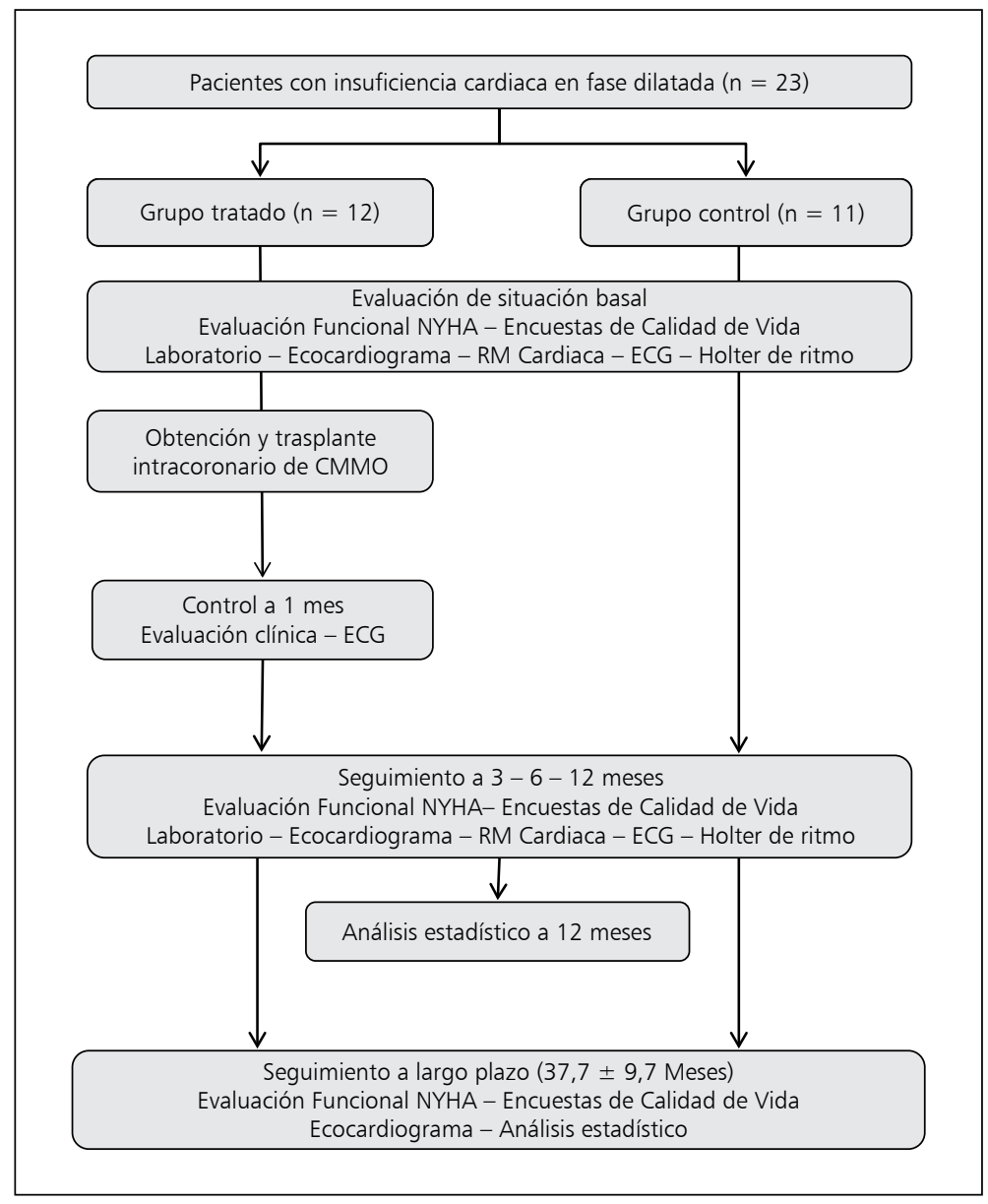

Figura 1. Diseño del ensayo clínico.

do criterios secundarios la capacidad funcional, según clasificación de la New York Heart Association (NYHA), los resultados de las encuestas de calidad de vida (descritas más abajo), y los volúmenes ventriculares evaluados por ecocardiografía.

\section{Ecocardiografía}

Las ecocardiografías transtorácicas fueron realizadas por dos cardiólogos, ciegos al tratamiento recibido por los pacientes, en un ecocardiógrafo Vivid 7 Dimension (General Electric, EEUU). La FEVI fue medida por Simpson modificado y se calcularon los volúmenes ventriculares izquierdos (telediastólico y telesistólico) en visión apical de dos y cuatro cámaras. Las mediciones de cavidades y el estudio de la función diastólica se realizaron de acuerdo a las guías de la Sociedad Americana de Ecocardiografía ${ }^{10}$.

\section{Encuestas de calidad de vida}

Los pacientes completaron traducciones lingüísticamente validadas de las encuestas Minnesota Living with Heart Failure Questionnaire (MLHFQ) y Kansas City Cardiomyopathy Questionnaire (KCCQ). El MLHFQ es un cuestionario auto-administrado de 21 ítems que busca medir el impacto de la insuficiencia cardiaca crónica sobre la vida del paciente, valorando aspectos físicos, socioeconómicos y psicológicos ${ }^{11}$. Las puntuaciones oscilan entre 0 y 105 , donde una puntuación más alta indica peor calidad de vida ${ }^{11}$. El KCCQ es un cuestionario auto-administrado de 23 ítems que aborda dominios específicos de salud relacionados con la insuficiencia cardiaca, incluyendo: limitación física, sintomatología, calidad de vida, interferencia social, autoeficacia ${ }^{12}$. Los primeros cuatro se combinan permitiendo un 
resumen clínico. Las puntuaciones varían entre 0 y 100 para cada dominio, donde puntuaciones más altas indican una menor carga sintomática y mejor calidad de vida ${ }^{12}$.

\section{Análisis estadístico}

Las variables continuas y categóricas se expresaron como promedio \pm desviación estándar o valor absoluto respectivamente. Se evaluó la normalidad de variables continuas mediante prueba de Shapiro-Wilk y consecuentemente fueron comparadas mediante prueba $t$ de Student o Mann-Whitney. Las variables categóricas fueron comparadas usando test de $\chi^{2}$. Para comparación de las evaluaciones ecocardiográficas, capacidad funcional y encuestas de calidad de vida, post tratamiento versus situación basal se empleó la prueba de Wilcoxon. Se consideró significativo un valor $\mathrm{p}<0,05$. Los análisis fueron realizados con el software IBM SPSS Statistics 19.

\section{Resultados}

\section{Características basales}

Las características basales de los pacientes se resumen en la Tabla 1, siendo discutidas en extenso en nuestra publicación anterior ${ }^{8}$. El único hecho a destacar, fue la mayor incidencia en el grupo tratado de disfunción diastólica grado III e insuficiencia mitral moderada o severa $(\mathrm{p}=0,004)$.

\section{Seguridad}

La incidencia de eventos clínicos mayores durante la totalidad del seguimiento se muestra en la Tabla 2. La mortalidad en el seguimiento fue similar en ambos grupos. Durante el primer año, un paciente de cada grupo falleció de muerte súbita. El paciente control falleció a los 17 días y el paciente del grupo trasplantado falleció a los 2 meses 27 días de ingresados al protocolo. En su evaluación inicial, ambos tenían arritmias ventriculares complejas en el Holter, bloqueo completo de rama izquierda con asincrónica septal y FEVI de $20 \%$. En el largo plazo, falleció un paciente de cada grupo producto de muerte súbita. El paciente del grupo control falleció a los 26 meses y 12 días de seguimiento, mientras el paciente tratado lo hizo a los 23 meses y 12 días de ingresado al protocolo. Ambos eran portadores de cardiopatía isquémica crónica, hemibloqueo izquierdo anterior y disfunción sistólica moderada al ingreso. El paciente tratado presentó un alza de 7 puntos de la FEVI a los 12 meses de seguimiento. Los síndromes coronarios agudos registrados correspondieron a cuadros de angina inestable. Ningún paciente presentó accidentes cerebrovasculares ni neoplasias. Los desfibriladores automáticos implantables fueron instalados bajo indicación de prevención primaria. Un paciente del grupo control se auto-excluyó del seguimiento a largo plazo, por traslado a una región distante del área metropolitana.

\section{Función ventricular}

En la Tabla 3 se expone la evolución de parámetros ecocardiográficos medidos. El grupo de pacientes tratados con CMMO autólogas no presentó un aumento significativo de la FEVI en el seguimiento a largo plazo respecto a basal $(+8,15 \pm$ $9,84, \mathrm{p}=0,059)$. No constatamos modificaciones significativas en los volúmenes ventriculares. En el grupo control no observamos cambios significativos en ninguna de estas variable ecocardiográficas.

\section{Capacidad funcional}

En la Tabla 4 se muestra la evolución en la capacidad funcional según NYHA de ambos grupos. En comparación al grupo control, el grupo tratado con CMMO presentó una peor capacidad funcional basal $(p<0,05)$. Los pacientes del grupo tratado presentaron una mejoría de la capacidad funcional durante el seguimiento a largo plazo ( $\mathrm{p}$ $<0,01)$. En el grupo control, no constatamos una mejoría significativa de la capacidad funcional durante el seguimiento ( $\mathrm{p}>0,05)$.

\section{Calidad de vida}

Los resultados de las encuestas de calidad de vida se muestran en la Tabla 4 . El grupo tratado presentó basalmente una mayor limitación física y social, así como un peor resumen clínico al ingreso según KCCQ $(p<0,05)$. Hubo una mejoría en la calidad de vida evaluada por ambas encuestas $(\mathrm{p}<0,01)$. Sólo el subdominio de autoeficacia de la encuesta KCCQ no resultó significativo. En el grupo control, se constató una mejoría del subdominio de calidad de vida del KCCQ $(\mathrm{p}<0,02)$, no demostrándose una mejoría sobre otros subdominios del KCCQ ni de la calidad de vida según MLHFQ (p > 0,05). 
Tabla 1. Características basales de las poblaciones de estudio

\begin{tabular}{|c|c|c|c|}
\hline Variable & Controles $(n=11)$ & Tratados $(n=12)$ & $\mathbf{p}$ \\
\hline Edad (años) & $57 \pm 11$ & $58 \pm 14$ & NS \\
\hline Hombres & $9(81,8 \%)$ & $8(66,7 \%)$ & NS \\
\hline IMC & $28,6 \pm 4,8$ & $28,2 \pm 4,9$ & NS \\
\hline Hipertensión arterial & $4(36,3 \%)$ & $4(33,3 \%)$ & NS \\
\hline Dislipidemia & $3(27,3 \%)$ & $1 \quad(8,3 \%)$ & NS \\
\hline Diabetes mellitus & $1 \quad(9,1 \%)$ & $2(16,7 \%)$ & NS \\
\hline Tabaquismo & $4(36,3 \%)$ & $3(25,0 \%)$ & NS \\
\hline Alcohol & $1 \quad(9,1 \%)$ & $2(16,7 \%)$ & NS \\
\hline Cocaína* & $1 \quad(9,1 \%)$ & $1 \quad(8,3 \%)$ & NS \\
\hline Quimioterapia $^{\dagger}$ & $0 \quad(0,0 \%)$ & $1 \quad(8,3 \%)$ & NS \\
\hline Aspirina & $7(63,6 \%)$ & $3(25,0 \%)$ & NS \\
\hline Clopidogrel & $2(18,2 \%)$ & $1 \quad(8,3 \%)$ & NS \\
\hline Acenocumarol & $7(63,6 \%)$ & $8(66,7 \%)$ & NS \\
\hline IECAVARA || & $9(81,8 \%)$ & $9(75,0 \%)$ & NS \\
\hline Beta bloqueador & $8(72,7 \%)$ & $10(83,3 \%)$ & NS \\
\hline Diuréticos & $10(90,9 \%)$ & $11(91,6 \%)$ & NS \\
\hline Digoxina & $3(27,3 \%)$ & $3(25,0 \%)$ & NS \\
\hline Vasodilatadores & $0 \quad(0,0 \%)$ & $3(25,0 \%)$ & NS \\
\hline Estatinas & $4(36,3 \%)$ & $4(33,3 \%)$ & NS \\
\hline Amiodarona & $1 \quad(9,1 \%)$ & $1 \quad(8,3 \%)$ & NS \\
\hline Miocardiopatía dilatada idiopática & $5(45,4 \%)$ & $8(66,7 \%)$ & NS \\
\hline Cardiopatía isquémica & $4(36,3 \%)$ & $2(16,7 \%)$ & NS \\
\hline Cardiopatía hipertensiva & $2(18,2 \%)$ & $2(16,7 \%)$ & NS \\
\hline Insuficiencia mitral moderada o severa & $2(18,2 \%)$ & $8(66,7 \%)$ & 0,004 \\
\hline Disfunción diastólica grado 3 & $2(18,2 \%)$ & $8(66,7 \%)$ & 0,004 \\
\hline Bloqueo completo de rama izquierda & $2(18,2 \%)$ & $3(25,0 \%)$ & NS \\
\hline Bloqueo completo de rama derecha & $2(18,2 \%)$ & $6(50,0 \%)$ & NS \\
\hline Hemibloqueo izquierdo anterior & $4(36,3 \%)$ & $6(50,0 \%)$ & NS \\
\hline Fibrilación auricular & $2(18,2 \%)$ & $1 \quad(8,3 \%)$ & NS \\
\hline Desfibrilador automático implantable & $1 \quad(9,1 \%)$ & $0 \quad(0,0 \%)$ & NS \\
\hline
\end{tabular}

*Consumo inveterado, suspendido $\geq 3$ años antes del ingreso al protocolo; 'Realizada en 1999 por leucemia linfoblástica, logrando remisión de la enfermedad, sin necesidad de otros tratamientos; IECA: inhibidor de enzima convertidora de angiotensina; ARA II: antagonista del receptor de angiotensina II, NS: no significativo. 
Tabla 2. Eventos mayores durante el seguimiento a largo plazo

\begin{tabular}{|lccc|}
\hline Evento & Controles $(\mathbf{n}=\mathbf{1 1})$ & Tratados (n = 12) & p \\
\hline Muerte cardiovascular & $2(18,2 \%)$ & $2(16,7 \%)$ & NS \\
\hline Muerte no cardiovascular & $0(0,0 \%)$ & $0(0,0 \%)$ & NS \\
Hospitalizaciones por ECV & $5(45,5 \%)$ & $2(16,7 \%)$ & $\mathrm{NS}$ \\
$\quad$ Insuficiencia cardiaca descompensada & $2(18,2 \%)$ & $2(16,7 \%)$ & $\mathrm{NS}$ \\
$\quad$ Síndrome coronario agudo & $2(18,2 \%)$ & $0(0,0 \%)$ & $\mathrm{NS}$ \\
$\quad$ Síncope & $1(9,1 \%)$ & $0(0,0 \%)$ & $\mathrm{NS}$ \\
\multicolumn{1}{|c|}{ Accidente cerebrovascular } & $0(0,0 \%)$ & $0(0,0 \%)$ & $\mathrm{NS}$ \\
Implante de dispositivos intracardiacos & $2(18,2 \%)$ & $0(0,0 \%)$ & NS \\
\hline
\end{tabular}

ECV: Enfermedades cardiovasculares; *Abandono después del primer año de seguimiento por traslado a una región distante del Área Metropolitana, NS: no significativo.

Tabla 3. Parámetros Ecocardiográficos: situación basal y seguimiento a largo plazo

\begin{tabular}{|c|c|c|c|c|c|c|}
\hline \multicolumn{4}{|c|}{ Controles } & \multicolumn{3}{|c|}{ Tratados } \\
\hline & $\begin{array}{l}\text { Basal } \\
(n=11)\end{array}$ & $\begin{array}{l}\text { Largo plazo } \\
\quad(n=8)\end{array}$ & $\mathbf{p}$ & $\begin{array}{c}\text { Basal } \\
(n=12)\end{array}$ & $\begin{array}{l}\text { Largo plazo } \\
\qquad(n=10)\end{array}$ & $\mathbf{p}$ \\
\hline FEVI & $30,3 \pm 6,3$ & $33,4 \pm 5,9$ & NS & $26,8 \pm 4,9$ & $34,9 \pm 8,6$ & NS \\
\hline VFS & $141 \pm 24$ & $146 \pm 61$ & NS & $194 \pm 74$ & $150 \pm 75$ & NS \\
\hline VFD & $207 \pm 33$ & $217 \pm 76$ & NS & $251 \pm 95$ & $202 \pm 75$ & NS \\
\hline
\end{tabular}

FEVI: Fracción de Eyección de Ventrículo Izquierdo; VFS: Volumen telesistólico; VFD: Volumen telediastólico. NS: no significativo.

Tabla 4. Capacidad funcional y resultados de encuestas de calidad de vida: situación basal y seguimiento a largo plazo

\begin{tabular}{|c|c|c|c|c|}
\hline & \multicolumn{2}{|c|}{ Controles } & \multicolumn{2}{|c|}{ Tratados } \\
\hline & $\begin{array}{c}\text { Basal } \\
(n=10)\end{array}$ & $\begin{array}{l}\text { Largo plazo } \\
\quad(n=8)\end{array}$ & $\begin{array}{c}\text { Basal } \\
(n=11)\end{array}$ & $\begin{array}{c}\text { Largo plazo } \\
(n=10)\end{array}$ \\
\hline NYHA & $2,13 \pm 0,50$ & $1,88 \pm 0,83$ & $2,63 \pm 0,57^{*}$ & $1,65 \pm 0,58 \ddagger$ \\
\hline MLHFQ & $54,0 \pm 23,6$ & $40,0 \pm 22,8$ & $65,6 \pm 26,4$ & $32,4 \pm 26,0^{\ddagger}$ \\
\hline \multicolumn{5}{|l|}{ KCCQ } \\
\hline LF & $62,5 \pm 35,1$ & $73,8 \pm 20,5$ & $32,7 \pm 24,8^{*}$ & $75,7 \pm 29,1^{\ddagger}$ \\
\hline ST & $49,0 \pm 28,6$ & $73,3 \pm 21,2$ & $31,1 \pm 25,4$ & $74,9 \pm 26,1^{\ddagger}$ \\
\hline CV & $44,8 \pm 27,7$ & $61,3 \pm 21,5 \ddagger$ & $21,2 \pm 25,6$ & $65,9 \pm 29,4^{\ddagger}$ \\
\hline IS & $68,8 \pm 27,3$ & $73,4 \pm 21,9$ & $32,5 \pm 33,5^{*}$ & $69,3 \pm 30,3^{\ddagger}$ \\
\hline $\mathrm{AE}$ & $55,0 \pm 18,8$ & $75,2 \pm 16,4$ & $64,0 \pm 33,3$ & $82,7 \pm 18,7$ \\
\hline$E F$ & $55,3 \pm 26,1$ & $73,4 \pm 19,0$ & $32,5 \pm 25,1$ & $75,5 \pm 27,3^{\ddagger}$ \\
\hline $\mathrm{RC}$ & $55,8 \pm 23,9$ & $72,0 \pm 19,4$ & $30,4 \pm 25,3^{*}$ & $72,9 \pm 26,7^{\ddagger}$ \\
\hline
\end{tabular}

NYHA: Capacidad funcional según clasificación de la New York Heart Association; MLHFQ: Minnesota Living with Heart Failure Questionnaire; KCCQ: Kansas City Cardiomyopathy Questionnaire; LF: Limitación física, ST: Síntomas; CV: Calidad de vida; IS: Interferencia social; AE: Autoeficacia; EF: Estado Funcional; RC: Resumen Clínico; ${ }^{\star} p<0,05$ comparado al grupo control; ${ }_{\mathrm{f}} \mathrm{p}<0,01$ comparado a situación basal. 


\section{Discusión}

\section{Seguridad de la terapia celular}

Ninguno de los pacientes sometidos a trasplante intracoronario de CMMO autólogas presentó complicaciones derivadas de este procedimiento a largo plazo, concordando con lo reportado previamente por otros grupos ${ }^{13-17}$. No se encontraron diferencias en la mortalidad o incidencia de otros eventos clínicos mayores entre ambos grupos en el seguimiento a largo plazo. La mayoría de las revisiones sistemáticas y estudios de meta-análisis han sugerido que la terapia celular no se asocia a un mayor riesgo de mortalidad, infarto agudo al miocardio, hospitalizaciones por patología cardiovascular, ni complicaciones relacionadas al procedimiento de trasplante, en pacientes con cardiopatía isquémica crónica o miocardiopatía dilatada no isquémica ${ }^{15,16}$. La terapia celular tampoco ha sido asociada anteriormente a una mayor incidencia de neoplasias ${ }^{18}$.

Ensayos clínicos randomizados con seguimientos a 5 años, tanto en pacientes con cardiopatía isquémica crónica como miocardiopatía dilatada idiopática, han descrito una disminución de la mortalidad cardiovascular en pacientes sometidos a terapia celular intracoronaria versus pacientes control $(\mathrm{p}<0,01)^{5,6}$. El último meta-análisis de terapia celular en pacientes con cardiopatía isquémica crónica también sugiere una reducción significativa de la mortalidad y la tasa de rehospitalización por insuficiencia cardiaca en pacientes tratados con CMMO en el largo plazo ( $\geq 12$ meses de seguimiento; $\mathrm{p}<0,05)^{17}$. Similar a nuestros resultados, el reporte final del ABCD, ensayo clínico realizado en pacientes con miocardiopatía dilatada idiopática, no describe diferencias en la sobrevida entre pacientes tratados y controles luego de 36 meses de seguimiento ${ }^{4}$. El beneficio en la sobrevida atribuido a la terapia celular en cardiópatas deberá ser confirmado por ensayos clínicos en fase III, actualmente en desarrollo (Clinicaltrials.gov NCT01693042, NCT02032004).

\section{Eficacia de la terapia celular}

No constatamos un beneficio persistente sobre la FEVI en pacientes tratados en el largo plazo. Tres ensayos clínicos, con una mayor cantidad de participantes que nuestro estudio, describieron previamente un aumento persistente de la FEVI en pacientes con cardiopatía isquémica crónica y miocardiopatía dilatada idiopática en seguimientos a 36 y 60 meses $^{4-6}$. En consideración a estos estudios, planteamos que nuestros resultados pudiesen estar condicionados por el pequeño número de pacientes, así como también por el fallecimiento por muerte súbita de uno del grupo tratado, quien presentó una respuesta favorable a la terapia celular durante el primer año, experimentando un alza de 7\% de la FEVI. En nuestro reporte anterior, describimos un aumento significativo de la FEVI a los 6 y 12 meses de seguimiento ${ }^{8}$. Existen algunas experiencias compatibles con nuestros resultados, en ensayos clínicos realizados en población con infarto agudo al miocardio tratados con CMMO, donde se señalaron beneficios en la FEVI limitados al primer año de seguimiento ${ }^{19,20}$. La FEVI como criterio de valoración de eficacia en protocolos de terapia celular ha sido parcialmente cuestionada, porque es dependiente de múltiples factores, incluyendo la precarga, la postcarga, la contractilidad miocárdica y la activación neurohumoral, entre otras ${ }^{21}$. Variables como la disminución del tamaño del infarto, dimensiones ventriculares o la mejoría de la función miocárdica regional, que pueden tener escaso impacto sobre la FEVI, han sido propuestas como mejores marcadores de eficacia de esta terapia ${ }^{21}$.

En nuestro estudio no se encontraron cambios significativos de los volúmenes ventriculares izquierdos, aunque si una tendencia a su disminución. La evidencia actual propone que el potencial impacto de la terapia con CMMO sobre el remodelado ventricular patológico es dependiente del proceso etiopatogénico de cada cardiopatía. Ensayos clínicos de terapia celular en pacientes con infarto agudo al miocardio o cardiopatía isquémica crónica han descrito reducciones sustanciales de los volúmenes ventriculares en sus seguimientos a largo plazo ${ }^{13-15}$. En contraste, el mayor estudio de terapia celular en pacientes con miocardiopatía dilatada idiopática, realizado por Vrtovec y cols., no demostró cambios significativos en de los volúmenes ventriculares en sus seguimientos a 12 y 60 meses $^{6}$. Nuestros resultados parecen consistentes con lo descrito por Vrtovec y cols., considerando la mayor prevalencia de miocardiopatía dilatada idiopática en nuestros participantes.

Nuestros hallazgos indican una mejoría de la capacidad funcional y la calidad de vida, evaluada mediante encuestas validadas, en el grupo tratado, durante la totalidad del seguimiento. Esto coincide 
con los resultados del estudio $\mathrm{ABCD}$, donde se describe una mejoría sustancial de la capacidad funcional por NYHA, así como el estado funcional y resumen clínico del $\mathrm{KCCQ}^{4}$. Otros estudios, en pacientes con cardiopatía isquémica crónica y miocardiopatía dilatada idiopática, han señalado beneficios modestos sobre la capacidad funcional y la calidad de vida evaluada mediante MLHFQ ${ }^{15,22}$.

\section{Limitaciones}

La principal limitación de nuestro estudio fue la falta de aleatorización, sin embargo, ambos grupos fueron semejantes. Una segunda limitación concierne al uso de ecocardiografía sobre la resonancia magnética cardiaca, siendo esta última actualmente considerada como método de elección para evaluar el remodelado ventricular en protocolos de terapia celular. Lamentablemente, ésta no puso ser realizada en todos nuestros pacientes por contraindicaciones para su uso, incluyendo presencia de dispositivos intracardiacos y claustrofobia. Finalmente, nuestro estudio presentó una muestra heterogénea de pacientes con cardiopatías de diferente etiología en fase dilatada. A pesar de este inconveniente, consideramos de gran valor la muestra seleccionada, pues es representativa de los pacientes que consultan habitualmente en los Servicios de Cardiología en nuestro medio.

\section{Conclusiones}

Este es el primer ensayo clínico latinoamericano que reporta resultados de terapia celular intracoronaria en pacientes con insuficiencia cardiaca, en un seguimiento a largo plazo. Nuestros resultados permiten concluir, en concordancia a lo descrito previamente, que el trasplante intracoronario con CMMO autólogas es un procedimiento seguro en pacientes con insuficiencia cardiaca. Adicionalmente, documentamos una mejoría persistente de la capacidad funcional y la calidad de vida de los pacientes tratados, en el largo plazo. Sin embargo, este hallazgo no se correlacionó con un aumento significativo de la FEVI en el grupo tratado, en el seguimiento a $37,7 \pm 9,7$ meses.

Agradecimientos: A los Servicios de Cardiología del Hospital San Juan de Dios, el Hospital Barros Luco Trudeau y la Clínica Santa María. Al Laboratorio de Terapia Celular de la Universidad de los Andes, Santiago de Chile. A INNOVA Chile, CORFO.

\section{Referencias}

1. Williams AR, Trachtenberg B, Velázquez DL, McNiece I, Altman P, Rouy D, et al. Intramyocardial stem cell injection in patients with ischemic cardiomyopathy: functional recovery and reverse remodeling. Circ Res 2011; 108 (7): 792-6.

2. Perin EC, Dohmann HF, Borojevic R, Silva SA, Sousa AL, Mesquita CT, et al. Transendocardial, autologous bone marrow cell transplantation for severe, chronic ischemic heart failure. Circulation 2003; 107 (18): 2294302.

3. Stamm C, Kleine HD, Choi YH, Dunkelmann S, Lauffs JA, Lorenzen B, et al. Intramyocardial delivery of CD133+ bone marrow cells and coronary artery bypass grafting for chronic ischemic heart disease: safety and efficacy studies. J Thorac Cardiovasc Surg 2007; 133 (3): 717-25.

4. Seth S, Bhargava B, Narang R, Ray R, Mohanty S, Gulati $\mathrm{G}$, et al. The ABCD (Autologous Bone Marrow Cells in Dilated Cardiomyopathy) trial a long-term follow-up study. J Am Coll Cardiol 2010; 55 (15): 1643-4.

5. Strauer BE, Yousef M, Schannwell CM. The acute and long-term effects of intracoronary Stem cell Transplantation in 191 patients with chronic heARt failure: the STAR-heart study. Eur J Heart Fail 2010; 12 (7): 721-9.

6. Vrtovec B, Poglajen G, Lezaic L, Sever M, Domanovic $\mathrm{D}$, Cernelc $\mathrm{P}$, et al. Effects of intracoronary CD34+ Stem cell transplantation in nonischemic dilated Cardiomyopathy patients: 5-Year Follow-Up. Circ Res 2013; 112 (1): 165-73.

7. Suárez de Lezo J, Herrera C, Romero M, Pan M, Suárez de Lezo J, Jr., Carmona MD, et al. Functional improvement in patients with dilated cardiomyopathy after the intracoronary infusion of autologous bone marrow mononuclear cells. Rev Esp Cardiol 2013; 66 (6): 450-7.

8. Bartolucci J, Carrión F, Lamich R, Pedreros P, Carvallo C, Sanhueza P, et al. Autologous bone marrow cell transplantation improves ejection fraction in patients with dilated cardiomyopathy of diverse etiologies. Proyecto INNOVA Chile No 205-4350. Rev Chil Cardiol 2013; 32 (1): 11-20.

9. Alfaro M, Asenjo R, Castro P, Jalil J, Llancaqueo M, Sepúlveda L, et al. Guías para el diagnóstico y tratamiento de la Insuficiencia Cardiaca. Rev Chil Cardiol 2007; 26 (4): 473-504.

10. Lang RM, Bierig M, Devereux RB, Flachskampf FA, Fos- 
ter E, Pellikka PA, et al. Recommendations for chamber quantification: a report from the American Society of Echocardiography's Guidelines and Standards Committee and the Chamber Quantification Writing Group, developed in conjunction with the European Association of Echocardiography, a branch of the European Society of Cardiology. J Am Soc Echocardiogr 2005; 18 (12): 1440-63.

11. Garín O, Soriano N, Ribera A, Ferrer M, Pont A, Alonso J, et al. Validation of the Spanish version of the Minnesota Living with Heart Failure Questionnaire. Rev Esp Cardiol 2008; 61 (3): 251-9.

12. Comin-Colet J, Garín O, Lupon J, Manito N, Crespo-Leiro MG, Gómez-Bueno M, et al. Validation of the Spanish version of the Kansas city cardiomyopathy questionnaire. Rev Esp Cardiol 2011; 64 (1): 51-8.

13. Clifford DM, Fisher SA, Brunskill SJ, Doree C, Mathur A, Watt S, et al. Stem cell treatment for acute myocardial infarction. Cochrane Database Syst Rev 2012; 2: CD006536.

14. Jeevanantham V, Butler M, Saad A, Abdel-Latif A, Zuba-Surma EK, Dawn B. Adult bone marrow cell therapy improves survival and induces long-term improvement in cardiac parameters: a systematic review and meta-analysis. Circulation 2012; 126 (5): 551-68.

15. Kandala J, Upadhyay GA, Pokushalov E, Wu S, Drachman DE, Singh JP. Meta-analysis of stem cell therapy in chronic ischemic cardiomyopathy. Am J Cardiol 2013; 112 (2): 217-25.

16. Gho JM, Kummeling GJ, Koudstaal S, Jansen OF, Lorkeers SJ, Doevendans PA, Asselbergs FW, et al. Cell therapy, a novel remedy for dilated cardiomyopathy? A systematic review. J Card Fail 2013; 19 (7): 494-502.

17. Fisher SA, Brunskill SJ, Doree C, Mathur A, Taggart DP, Martin-Rendon E. Stem cell therapy for chronic ischaemic heart disease and congestive heart failure. Cochrane Database Syst Rev 2014; 4: CD007888.

18. Lalu MM, McIntyre L, Pugliese C, Fergusson D, Winston BW, Marshall JC, et al. Safety of cell therapy with mesenchymal stromal cells (SafeCell): a systematic review and meta-analysis of clinical trials. PLoS One 2012; 7 (10): e47559.

19. Beitnes JO, Gjesdal O, Lunde K, Solheim S, Edvardsen $\mathrm{T}$, Arnesen $\mathrm{H}$, et al. Left ventricular systolic and diastolic function improve after acute myocardial infarction treated with acute percutaneous coronary intervention, but are not influenced by intracoronary injection of autologous mononuclear bone marrow cells: a 3 year serial echocardiographic sub-study of the randomized-controlled ASTAMI study. Eur J Echocardiogr 2011; 12: 98-106.

20. Assmus B, Rolf A, Erbs S, Elsasser A, Haberbosch W, Hambrecht R, et al. Clinical outcome 2 years after intracoronary administration of bone marrow-derived progenitor cells in acute myocardial infarction. Circ Heart Fail 2010; 3 (1): 89-96.

21. Suncion VY, Schulman IH, Hare JM. Concise review: the role of clinical trials in deciphering mechanisms of action of cardiac cell-based therapy. Stem Cells Transl Med 2012; 1 (1): 29-35.

22. Martino HF, Oliveira PS, Souza FC, Costa PC, Assuncao ESE, Villela R, et al. A safety and feasibility study of cell therapy in dilated cardiomyopathy. Braz J Med Biol Res 2010; 43 (10): 989-95. 